\title{
Planet-hunters seek cheap missions
}

\section{WASHINGTON DC}

Since NASA put its Terrestrial Planet Finder (TPF) missions on indefinite hold earlier this year, researchers have proposed at least four alternative and much cheaper spacecraft that could capture starlight reflected from other worlds beyond our Solar System.

The proposals range from using a giant shield placed 1.5 million kilometres from Earth, as suggested by Webster Cash of the University of Colorado, Boulder, in this week's Nature (see page 51), to telescopes with unusual mirrors and finely engineered cameras. The missions have been proposed to NASA's Discovery programme, which caps the costs of its projects at US $\$ 425$ million - about a tenth of the cost of NASA's successor to Hubble, the James Webb Space Telescope (JWST).

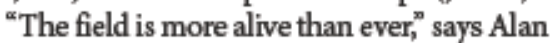
Boss, a planetary modeller at the Carnegie Institution of Washington.

Astronomers have indirectly detected 194 extrasolar planets so far, all from the ground, by watching the behaviour of their parent stars. But for direct detection, and to stand a chance of studying the planets detected, orbit is the place to be.

The TPF, which would cost much more than the JWST, consisted of two planet-spotting missions that would have picked up small fry the size of Earth. The mission was among the top recommendations of a 2000 National Research Council panel, and featured in President
George W. Bush's vision for space exploration in 2004. But competing priorities caused the agency to decide that the TPF should be "deferred indefinitely" and to delay a smaller planet-hunting Space Interferometry Mission by three years (see Nature 439, 768-769; 2006). A similarly ambitious European mission, Darwin, has a proposed launch date of 2015.

But researchers are increasingly optimistic that they can glimpse an alien planet through other methods.

Cash's scheme is to place a specially designed piece of black plasticbag-like material, 30-50 metres in diameter, between the JWST and a target star, allowing the scope to pick up the faint light of orbiting planets. The shield would have to be about 40,000 kilometres
"If we were relying solely on NASA space missions, the field would have screeched to a halt." been an exponential growth in concepts," says Olivier Guyon, an astronomer at the Subaru telescope on Mauna Kea, Hawaii. Guyon has his own application in with NASA for a 1.2-metre space telescope, which would have a configuration of aspherical mirrors that cut down the light from a star enough to directly observe Jupiter-like planets in nearby systems.

Other teams from NASA laboratories have their own ideas. The Eclipse mission from the Jet Propulsion Laboratory in Pasadena, California, would use an advanced camera to screen out starlight, while the EPIC mission from the Goddard Space Flight Center in Greenbelt, Maryland, would use interferometry to cancel out starlight, leaving dimmer planets visible. Richard Lyon, EPIC's project scientist at Goddard, says that if his from the telescope - itself around 1.5 million kilometres from Earth - and designed in a distinctive petal shape so that starlight leaking around the edges doesn't drown out the planets. It would have to be able to relocate over great distances to interpose itself between the JWST and various different stars, making it

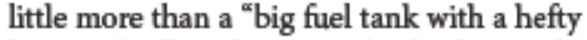
bag attached", Cash says. But he thinks it could reveal Earth-sized planets around nearby stars.

NASA is now considering Cash's scheme, along with several other ideas, as possible Discovery missions. "In the past five years, there's

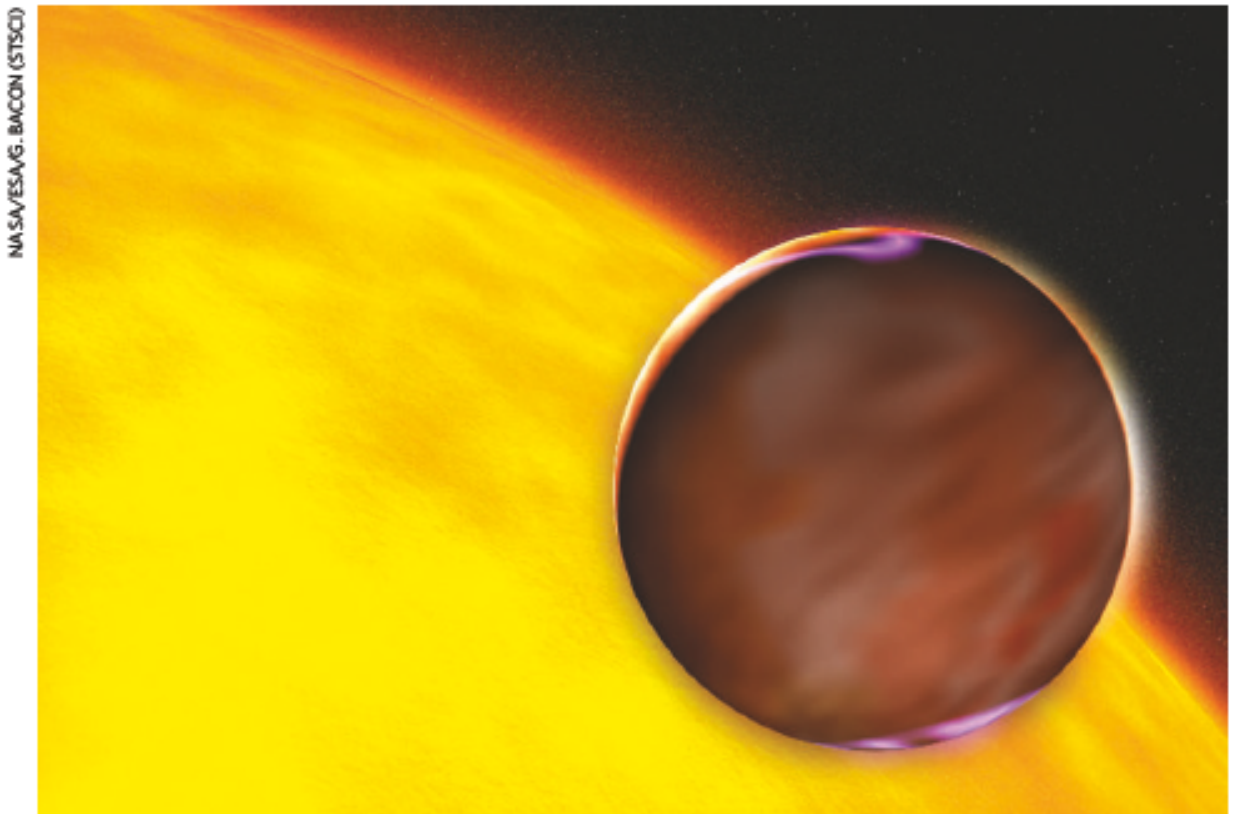

Hope is growing that Earth-like planets beyond our Solar System might be detected by new methods. team does not win funding this autumn, they might be able to make the mission even cheaper by shrinking the telescope to fit inside a 1.5metre-long tube just 45 centimetres in diameter. But a telescope that small, he notes, would have to stare at a single star for almost a year before gathering enough light to detect a planet.

It's not certain that any of these missions will reach space, says Boss. Because of cutbacks to NASA's science budget, researchers must apply for grants for these missions through the Discovery programme, which is normally reserved for missions within our Solar System. Only one such project - the Kepler mission to search for Earth-sized planets eclipsing distant stars - has won funding that way. Kepler, a 1.4-metre telescope, is set to launch in 2008.

Now TPF itself may have been granted a small reprieve, according to Charles Beichman, the mission's project scientist at the Jet Propulsion Laboratory. A congressional budget committee has tentatively earmarked $\$ 10$ million for the programme, after the president's budget request earlier this year would have wiped it out completely. If finalized, the money would keep the mission on life support. "But it's clear that the TPF is going to be a long time in the future," says Beichman.

Meanwhile, several ground-based searches are in the planning stages or under way. And in October, the French space agency plans to launch a 30-centimetre space telescope called Corot, with a mission similar to Kepler's. "If we were relying solely on [approved] NASA space missions, the field would have screeched to a halt," says Boss. "But thank God we're not." GeoffBrumfiel 